\title{
Update on the In-Training Exam
}

For more than 25 years, the In-Training-Examination (ITE) of the American Board of Family Medicine $(\mathrm{ABFM})$ has been a valuable resource for residency programs. An unusual aspect of this examination is that since 1981, an international program at the American University of Beirut has administered the ITE to its residents on the same day that it was given to residents in the United States. Although Beirut's residents were not included in the scoring statistics, they received the same score reports as their American counterparts, allowing them to compare their performance to US residency programs.

In 2006, the ABFM introduced an Internetbased version of the ITE in a pilot project. This allowed the ABFM to expand the use of the ITE to other international programs. In Australia, physicians from the Australian College of Rural and Remote Medicine (ACRRM) were able to take the ITE. In addition, Dr. Chris Place, Program Director of the Hope Family Medicine Residency Program in Macao, The People's Republic of China, worked with the ABFM to have his residents take the examination. The success of the pilot project both in the United States and also with the 2 international programs has resulted in the ABFM expanding the Internet-based delivery of the ITE, using a large commercial testing organization to administer it. In 2007, it is expected that additional international programs will take advantage of the Internet-based version. Dr. Timothy Fader of the Family Medicine Residency Program of Kabul, Afghanistan, and Dr. Roy Ringenberg of the Family Medicine Residency Program of Quito, Ecuador, are working with the ABFM to have their residents take the ITE in 2007.

Given the interest of international groups in the ITE, the ABFM is working with a number of organizations and individuals to improve the process by developing translated versions of the examination. Although these efforts are currently focused on individual volunteers, the ABFM hopes to obtain funding to support these activities in the future. 